\title{
Electronic Communication: Themes from a Case Study of the Deaf Community
}

\author{
Valerie Henderson-Summet ${ }^{1}$, Rebecca E. Grinter ${ }^{1}$, \\ Jennie Carroll ${ }^{2}$, and Thad Starner ${ }^{1}$ \\ ${ }^{1}$ Georgia Institute of Technology, GVU Center, Atlanta, GA, USA \\ $\{$ vlh, beki, thad $@ @ c c$. gatech.edu \\ ${ }^{2}$ University of Sydney, Business Information Systems, NSW, Australia \\ j.carroll@econ. usyd.edu. au
}

\begin{abstract}
We present a qualitative, exploratory study to examine the space of electronic communication (e.g. instant messaging, short message service, email) by Deaf teenagers in the greater Atlanta metro area. We discuss differences and similarities between deaf and hearing teen's usage of electronic communication mediums. Five common themes: Identity, Connection, Control, Tension, and Convenience were identified from the analysis of the data collected. These themes allow us to explore electronic communication from the "use-centric" view of teenagers who are indifferent to the underlying technology supporting this communication.
\end{abstract}

\section{Introduction}

In 1982 Barbara Wagreich, a deaf-blind computer professional wrote an article about the possibilities of a new technology, email, and how it might prove beneficial for people with disabilities, particularly the deaf [23]. Her study, conducted from 1978-1981 with deaf participants, their friends, and their families, showed that email was not only a formal medium for business meetings and communications, but also an informal tool for maintaining and furthering friendships. Twenty-four years later email is still used in this fashion but is complemented by the technologies of Instant Messaging (IM) and Short Message Service (SMS) or text messaging. These technologies have been widely adopted by the hearing population [16, 12, in particular by teenagers [19,8, 7. However, it is unclear how these new methods of communication are being used by the Deaf community 1 who are often reported to be early adopters of technologies [18, 10, 1, and there is very little formalized work studying the use by the Deaf community.

\footnotetext{
${ }^{1}$ The word "deaf" can have several meanings. Medical deafness focuses on the severity and cause of a hearing loss and is denoted with a lowercase "d,' "deaf." The cultural definition of Deaf, with an uppercase ' $\mathrm{D}$ ', is a voluntary classification and refers to the community formed by individuals whose primary method of communication is ASL. All the participants of this study were deaf, and most identified themselves as Deaf which is how I refer to them collectively in this paper.
} 
This work begins to fill this void with an exploratory study to understand the uniqueness of Deaf teenagers' electronic communication and to suggest further research directions.

We begin with related work on teenage communication patterns, and then discuss our study, the participants, and the data obtained. This includes an in-depth look at a variety of technologies the teens used. We also discuss the technology infrastructure in the United States and how it affects the communication choices Deaf teens make. We then describe the five themes that were identified through our analysis of the data collected from the multi-method study.

\section{Related Work}

In their work on SMS, Barkhuus and Vallgårda found that SMS was used to communicate mainly among friends and significant others, but IM was used for a wider range of conversational partners [2. Grinter and Eldgridge found that teens adopted mobile messaging for a variety of reasons, prominently, to coordinate conversations via another medium [7,8]. In a market report on mobile technologies, Blinkoff and Barranca found three central themes which users wanted: manage relationships, experience the unexpected, and avoid mobile stress [5]. In a study on teenage communication preferences, Schiano et al. found that home phones were the most common communication medium 19. Much of this work points to electronic communication from mobile devices (usually SMS) as being a transition medium which is used to coordinate voice communications via mobile or "landline" phones. This usage pattern points to a reliance on the voice telephony capabilities which many deaf individuals do not use, and this situation presents a significant difference between hearing and deaf teenagers.

While it might seem that work surveying the use of electronic communication methods among hearing teenagers [7,8, 19, would be sufficient to understand Deaf teens' communication, there are distinct linguistic differences between hearing and Deaf teens which warrant further exploration. For many people who are born deaf in the United States, their native language is American Sign Language (ASL). 2 Unlike English, ASL does not have a written form. It is languages' written form which enables electronic communication such as SMS, IM, email, etc. to exist. Deaf reliance on a medium which requires the use of a foreign language seems improbable. However, Bakken [1] found that Deaf teens in Norway relied on SMS messaging for building social networks, maintaining those networks, and for keeping abreast of trends and gossip. However, Bakken's work may not generalize to the United States population due to the differences between Europe and the US in SMS use and mobile device adoption.

It is also worthwhile to understand some of the linguistic issues which can arise from being born deaf in the United States. Linguists have identified the existence of a "critical period" for language development - a period during which a child

\footnotetext{
${ }^{2}$ ASL, the dominate sign language of North America, is a spatial, gesture based language which uses different hand, face, and body gestures to communicate. ASL's grammar is different from English. For a more complete discussion of ASL, see 22.
} 
must be exposed to and immersed in any language, including ASL [15, 17] to further linguistic development. The slower linguistic development of deaf children has been attributed to incomplete language models and lack of daily interaction using a language 21] unless their parents are also deaf and can sign to them from birth. These issues contribute to the fact that the average 17-18 year old deaf student reads at a 9-10 year old level [11.

Given the different characteristics of hearing and Deaf teenagers, we designed a research study to investigate the different ways in which electronic communication technologies were used in the Deaf community. Having introduced work on communication by teenagers, we now to turn to a description of study methods, participants, and methodological issues.

\section{Study Design and Methodology}

We recruited twelve participants with the cooperation of the Atlanta Area School for the Deaf (AASD). This is a public school for students who are deaf, and its enrollment area covers the majority of North Georgia, including the Atlanta metro area. On average, the students lived almost 30 miles from AASD. The maximum distance between students was almost 100 miles. This distance often precluded the teenagers from associating with their social community when not attending school. The students had a strong community at school; however, these students often cannot communicate easily with the hearing teens in their neighborhood or local hang-out spots. Because of this, they often feel isolated.

The twelve participants ranged in age from 14-17 with an average age of 16 . There were six females and six males, although one male student left the study after the first activity. Several of the students had some residual hearing, but not enough to make a school with auditory instruction feasible. Four students had enough residual hearing as to have some speech and to use oral communication with some degree of success.

The study had three phases designed to survey different aspects of the role of electronic communication in Deaf teenager's lives. The three phases were a social mapping activity, a diary study, and discussion groups, and these are described in more detail below.

Social Mapping Activity: The social mapping activity was designed to elicit the teenagers' social networks and give us an idea who they communicated with, whether those people were hearing or deaf, and the main techniques for communicating with those people.

Based upon the work of Smith, et.al 20, the teens were given a large sheet of paper and pencils. They were first asked to list everyone with whom they felt it was important to communicate. The definition of "important" was left to the participants, but we asked them to consider if they would be upset or unhappy if they could not communicate with a person. Second, the students were asked to label each person on their list as "hearing" or "deaf." After that, they were asked to go through their list and write down how they would communicate with that person in two different situations: face-to-face and remotely. They were asked 


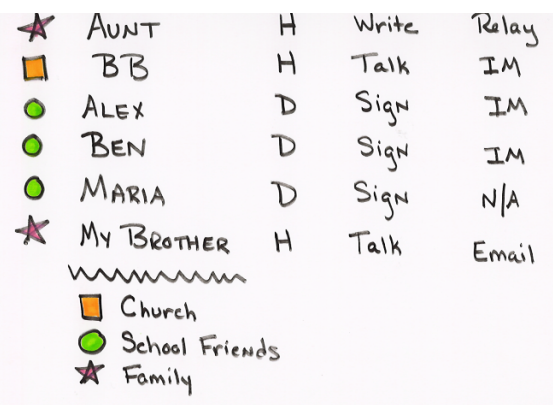

(a) Social Network

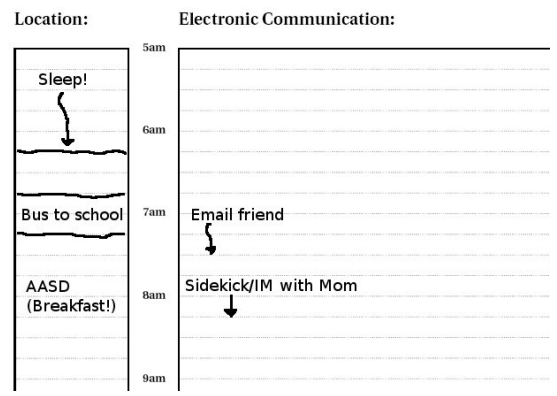

(b) Diary Form

Fig. 1. Examples of a Social Network and Diary Study Form

to write N/A or "Nothing" if they did not communicate with a contact. Lastly, the students were asked to group and categorize their contacts in any way they felt appropriate. Figure 1a shows an example of a social network similar to those generated by the students, with the contacts and their characteristics listed in the top half of the figure and the categories listed on the bottom.

Diary Study: The diary study phase involved students recording when and where they used electronic communication. Based on the work of Grinter and Eldridge [8, 7, it was designed to give a clear picture of the teenagers' lives on a daily basis and how electronic communication fit into it.

This form contained space to record one day's activities per page. Each hour was subdivided into 4 15-minute blocks, and the students were asked to record the location and nature of their communication. Figure $1 \mathrm{~b}$ gives an example of the form the students used. The students were given a booklet containing 7 forms, one for each day for a week.

Discussion Groups: After the social mapping activity and week-long diary activity, the students were interviewed. The interviews were conducted in two groups based on the student's class sections. Thus, they were with people they already knew and with whom they were comfortable talking. The interview questions were largely determined using data from the social maps and weekly diaries. We also allowed the students to highlight other topics they felt relevant.

After collecting this data we coded it according to several different schemes. We first coded the interview data by device used and looked for patterns and themes that were specific to a particular technology. We then recoded the data by more general categories such as "Who, what, when, where, why?" and looked for themes which emerged regardless of the device or technology used. We then used data from the social maps and journals to help validate or reject our emerging themes. The preliminary findings were reviewed by external researchers for further validation. 


\section{Results}

Originally, our study set out to examine the overall space of electronic communication use. However, it soon became clear that the majority of the teens used some kind of mobile device and the data naturally reflected that fact.

In this section, we discuss the preferred methods of communication used by the teens. We then discuss communication infrastructure in the United States and how this affects the teens' choices. In Section 5, we then present five central themes of communication that we extracted from our data and present the teens' communication within the framework of those themes.

Electronic communication preferences: As email was to Wagreich in 1982, the mobile platform has become to Deaf teens today. It levels the playing field by lowering the barrier of participation and allows them to maintain their friendships in ways that are convenient and simple. When students needed to communicate with someone they knew to be nearby, they would usually seek them out in person. However, when unsure of a person's location or when the person was far away, the students usually turned to a lightweight electronic method of communication rather than the more traditional method of relay or TTY 3 The teens we interviewed overwhelmingly favored establishing electronic means of communication after meeting new people. Most expressed a preference for exchanging email addresses or IM screen names. One student noted that many hearing people asked for an email address or a phone number for text messaging. Since he could never remember his phone number, he simply gave them his email address instead.

Devices and Infrastructures: Mobile text messaging has been increasing in popularity in the United States, but has never achieved the widespread acceptance seen in Europe or parts of Asia 12. Few teenagers pay for their own phone usage or use "pay as you go" plans. Text messages are not included in standard mobile service plans, and providers in the US often charge both the sender and the receiver of text messages and voice calls. Additionally, most providers provide "free night and weekend" plans which allow free voice calls after 9pm on weekdays and all day on weekends. This leads many hearing teens to wait until free calling periods and not use text messaging.

The most prized mobile device among the Deaf teenagers was clearly the T-Mobile Sidekick 4 Many of the teens already owned this device, and some

${ }^{3}$ TTYs (also called teletypewriters or TDDs) have been an accepted way for deaf individuals to communicate. If each person has a TTY, they can type messages back and forth using TTYs connected to standard telephone lines. To communicate with someone who does not own a TTY requires a third party relay operator.

${ }^{4}$ The Sidekick is a device marketed in the US by the service provider T-Mobile. It is designed as an out-of-the-box Internet platform with a mini-QWERTY keyboard. It includes software for web browsing, instant messaging, email, address book, and SMS. T-Mobile also offers unlimited, data-only service for this device, making it attractive to deaf students who do not need the voice capabilities. 
expressed a desire to upgrade, and those that did not have one expressed a desire to own one. However, none of them paid for it themselves. The Sidekick has become so ingrained in the teens' lives that it has a unique sign in ASL which mimics the screen popping up on the device. Only one student with verbal abilities used a mobile phone for voice telephony.

Some of the students in our study did not have a mobile device, but did have a family computer. Several students with mobile devices commented that they disliked computers. Two specific complaints were that "you're stuck in one place" and that computers had "a lot of things going on." Interestingly, one student noted that a legacy technology kept him tied to the computer. He had an old screen name through a service which did not work on his mobile device. He used his old screen name on the computer and his new one on his mobile device. The use of the computer for communication was drastically different from the use of mobile devices. Students reported only occasional, not constant communication via IM or email from a computer. Like Schiano's findings 19, the teens did not mode switch to email once they were online and chatting via IM. When they did use email, they responded to emails as soon as they received them, but complained that email was much slower than IM and "it takes a whole day to get it maybe."

\section{Themes of Communication}

We coded the interview portions of the study using open or inductive coding [14. Combining this data with analysis of the social maps and diary studies, five clear themes emerged. To the teenagers, communication is: Identity, Connection, Control, Tension, and Convenient. These themes are pervasive in the teens' communication. They are useful to designers because they emphasize the importance of the central purpose rather than the technological specifics of devices, communication methods, protocols, and other issues that often influence design decisions.

\subsection{Communication Is Identity}

The teenagers viewed their electronic communication as a vital piece of their identity. They also manipulated and managed the identity they created online. In certain circumstances the teens used their communication to rebel by communicating in times or places when it was forbidden. In some cases, while unable to detect the noise it made the teens were aware that they needed to mute their device to avoid detection. The vibrate setting was the de facto alert mechanism for most teens, but they were aware that even that could occasionally be detected. When asked why they went to such lengths to avoid detection, one student summed up her feelings as, "I don't want everyone looking at me." The style of communication allowed her to preserve her privacy. They were aware of how the communication affected others around them and might reflect on them in public. 
Students had no qualms about IMing someone not co-present even while physically with someone else. The teens didn't consider it rude if someone they were with also messaged other people. Their diaries often showed that they were collocated with members of their social circle (at dinner with family, for example), but IMing with friends via the mobile device. However, the teens felt that messaging should be conducted during breaks or lulls in the conversation. Being kept waiting to chat face-to-face by someone messaging was "wasting my time." Messaging while collocated was seen as something to fill time when their conversational partner was distracted by talking or driving. One participant noted, "You know sometimes, like with hearing people, they'll be talking to someone, and I feel left out. So I IM my sweetheart." Her communication usage allowed her to feel included even when she was with people who excluded her.

Somewhat surprisingly, given their difficulty with written English, most students expressed only minor worries about grammar or spelling mistakes. These mistakes were considered inconsequential for the most part, particularly among friends. One student said, "If I don't know how to spell it, I just make it up" while another noted, "Sometimes, if I get the grammar wrong or whatever, I'll just send [the message] anyway." One participant said he would generally ask his mother for help, but several others relied on the Sidekick, noting that it had built in spell check and grammar help, for example adding an apostrophe to a student's spelling of "Ive" instead of "I've."

\subsection{Communication Is Connection}

All the students considered communication with hearing friends and relatives to be an important component of their lives. Some students saw a mobile device as a means to enable that connection. The method of communication was less important than the ability to convey meaning and establish connections. In the words of one student, "The important thing is that people understand what I'm saying."

Like Barkhuus and Nardi [2, 16, we found that the primary recipients of IMs were the Deaf teens' friends, and they valued this ability to communicate very highly. They mostly reported messaging people who were not co-present, as they preferred to communicate directly with collocated people. One notable exception was a student who told us she used her Sidekick with a hearing person, passing the device back and forth. "When I can't hear [people], but they don't know how to sign, it's the only way we can actually get the point across." Her electronic communication allowed her to easily establish a connection with people she would not have been able to otherwise.

The students also reported IMing from the Sidekick to maintain connections with groups of their friends in large, multi-user sessions. Some of the students clearly enjoyed the large conversations with "lots of chatting going on." However some students were indifferent or clearly blasé about group conversations. Several students said it depended on what was going on. Another student noted that it could be "kinda annoying." Yet another characterized group IM conversations as "Blah, blah, blah." 
One student described using away messages as a social activity, maintaining a large buddy list and reading away messages because she was curious what people were doing. Grinter et al. and Nardi found a similar use of IM for awareness in their studies of IM [9, 16]. While not a turn-taking form of communication, the student was still maintaining connections with her peers and awareness of their activities.

The data from the social maps also demonstrates how dependent the teens have become on staying in touch via electronic methods. When analyzing this data, we found it interesting to examine the difference in how the teenagers communicated with their hearing and deaf friends, both in face-to-face communication and when not collocated. Figure 2 shows a graph of the data obtained from the teenagers' social maps. The top node shows the total of 419 contacts the teens listed in their maps. These were broken into three main categories based on the students' contacts' hearing ability: Hearing Contacts, Deaf Contacts, and Hard of Hearing or Unknown. Each of these categories were further split into methods of communication: Face-to-Face and Remote. The methods of communication were then listed in order of preference. For example, Figure 2 shows that 201 of the 419 contacts were deaf. For 190 of those 201 contacts, ASL was the preferred method for face-to-face communication, and for 124 of 201 contacts, IM was the preferred method of remote communication.

While this data should not be generalized due to the limited number of participants, several interesting things can be seen from this chart. An interesting trend is the differences in remote communication methods between the students' deaf contacts and hearing contacts. IM is the preferred communication method between the Deaf teenagers and other deaf people, used for 124 of 201 contacts. However, there is no clear preference for their hearing contacts who are not collocated, with the Deaf teens having no remote contact with $31 \%$ (68 out of 214) of hearing contacts and email being the preferred method of only $32 \%$ (64 out of 214 ).

The category of friends which the students do not maintain face-to-face relationships with (i.e. the "None" classification under the four face-to-face communication paths in Figure 2) also bears closer examination. There were 39 contacts that the students listed that they did not have any face-to-face communication with regardless of hearing status. However, the students communicated with 36 out of those 39 people remotely via the electronic methods of email and IM. (Of the other 3 people, 2 listed no communication either face-to-face or remotely and were obvious aberrant data points; the other wrote letters.) Before electronic communication existed and was widely available, remote communication with a person you had not met face-to-face would have taken the form of written letters (i.e. "pen-pals"). However, the teens today are using email and IM to do the same thing but with faster and more synchronous communication.

\subsection{Communication Is Control}

The teens' usage of communication also showed how they used it to feel more in control of their lives. An "easy" way to communicate clearly made the teens feel safer which appeared to be a key component of feeling in control. 
The students controlled their contacts in a variety of ways, including blocking and multiple screen names. Blocking is a standard feature of most IM clients and allows users to block messages from other users. One student noted she usually only blocked advertisements and spam. Another volunteered that he never blocked anyone. Several would immediately block someone they didn't know, but one student would try to talk with people before blocking them, explaining, "Maybe it's a new friend. I wouldn't mind trying to talk to them." However, that student went on to state she would block them if she did not feel comfortable with them. Students would also block people who simply annoyed them either through the content of messages or the volume of messages sent. They would block friends if they were having a fight or disagreement. Although some students reported that acquaintances they blocked were quite upset, they still utilized the feature. One student noted that she would unblock acquaintances after some time to see if they still bothered her.

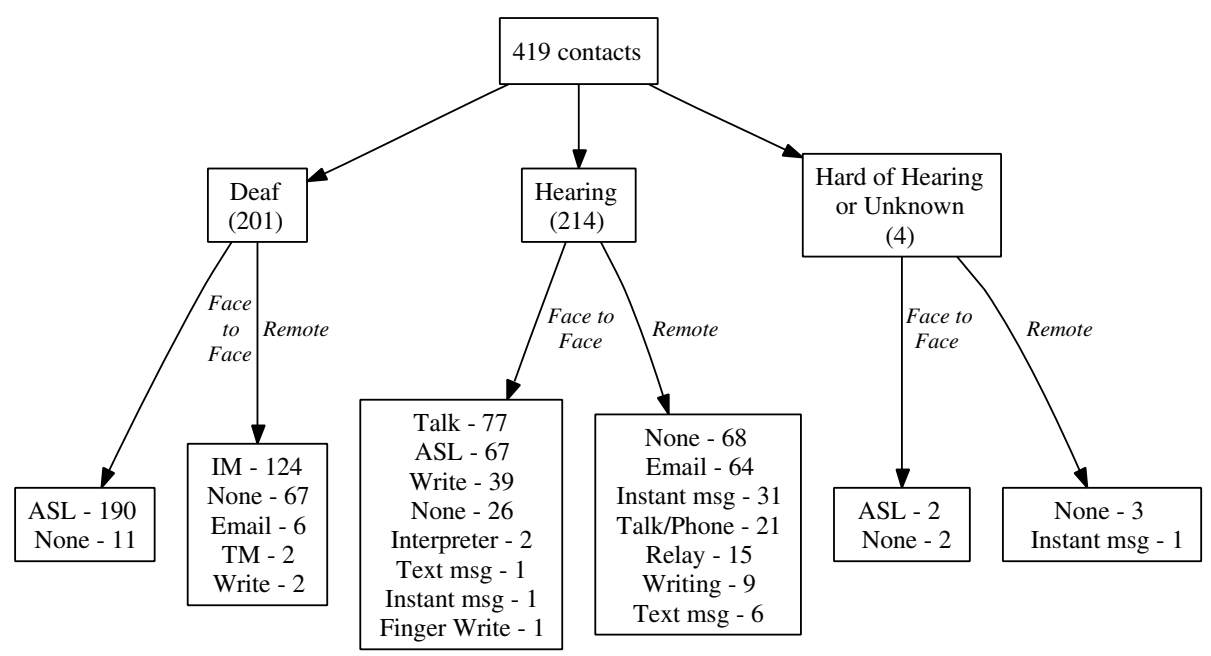

Fig. 2. Results for Social Networking Study

While not a specific medium of communication, away messages filled an important aspect in the teenager's communication spectrum, just as Baron found in her work on college students use of away messages [4]. The teens used a variety of away messages to control the flow of communication and indicate availability. Many students left a time estimating when they would return in their away messages before going out or being involved in other activities. They went to great lengths to maintain an accurate away message, including minute by minute updates. "When I get up in the morning, I immediately have to change [my away message] to my 'Hi, I'm at school now' message. I don't want people to think I'm sleeping then!" The away message removed the obligation of an immediate response. 


\subsection{Communication Is Tension}

The communication usage also raised tension in the teens' lives. While they loved the positive aspects it provided, it also added negative artifacts to their lives.

Unlike other studies of hearing teenagers [13, very few of the students reported that their parents used the device as a tool to enforce discipline. Most students reported that their parents put few or no restrictions on the use of the device at home, including use during mealtimes or curfews. Six students reported that their parents didn't restrict their use at all, while another 2 reported some restrictions such as not using the device after a certain time on school nights or putting it away at mealtimes. One student pointed out, "Mom is fine because she knows, like, I'm Deaf and I want to communicate with people. And she knows it's OK. It's not wasting our time." One teen's parents would not allow him to have one due to the expense and possibility of losing it but used the device as incentive, hinting he might get one if he kept his grades up.

The school, however, banned the use of the devices. Students were not allowed to use the device during class hours and were instead limited to breakfast or lunch times. The rule had recently been strengthened, requiring parents to come and pick up the device if it was confiscated. Since these rules had been implemented, several students reported they had gotten in trouble for using the device at school. In fact, many told us that they now left their Sidekicks at home to avoid the temptation. However, the students who regularly carried their devices noted that any time without the device felt strange.

Tension also arose from the ease and prevalence of communication availability. Like the hearing teens in Smith et al.'s study 20, the Deaf teenagers sometimes felt overwhelmed by the sheer numbers of contacts and the social energy expended maintaining them. One student reported that having more than one screen name was "too much to keep up with." In contrast, another told us that she would simply make up a new screen name when her current one hit the maximum number of contacts allowed (around 200 contacts by her estimation.) Other students reported 20,89, and 72 people on their buddy lists. One student noted that having many people on her buddy list led to people contacting her constantly which "gets kinda silly at times."

\subsection{Communication Is Convenient}

The students viewed their communication as highly convenient. It was clear that the device preferences and communication modes arose due to the convenience it afforded the users. The freedom of a personal platform was greatly valued and this freedom was exercised in a variety of ways.

The students used communication via mobile devices heavily. Most students who had a device reported using it daily with a majority of the use occurring during the free time after school or on the weekends. In their daily diaries, students reported several hour-long blocks of IM without interruptions. When questioned, the students assured us that was correct and that they were constantly chatting. One student observed, "I [chat] a lot. Even in my sleep I do 
it." One student admitted he chatted with his friends all day, saying his practice was, "kinda lazy, I guess."

In addition to just chatting, IM was viewed as an optimal, convenient way to schedule things with friends. This mirrored findings by Nardi et al. and Grinter and Palen [16, 9] that the immediacy of IM was useful for coordination and scheduling. Scheduling things was of great importance to the teens given their lack of transportation and distance from friends. The teens could refine their plans on-the-fly, but plans were usually made in advance due to the logistics of meeting. Only if a friend was offline was email employed to schedule things. Even then, email was usually used to establish a time when both would be available to IM and finalize the details.

The students choice of electronic communication medium also reflected the teens' desire for convenience. For example, most students preferred IM and saw text messaging as a backup communication medium (to be used only as a last resort). Text messaging was something to be used only if the other person lacked some vital functionality. The students might use text messaging if someone didn't have IM or email, if a friend wasn't online, or if a friend wasn't in a position to check email. Cost was not a deciding factor, unlike Grinter's study [7] which found that teenagers made a determination based on cost $27 \%$ of the time. This can be explained by the fact that none of the teenagers in our study paid for their own costs and the unlimited data plan that many of them had. One participant identified text messaging as a feature central to mobile phones and said that she used text messaging because most other people had a cell phone thus increasing the number of people with whom she could communicate. Another posited that he might use text messaging only in an emergency situation.

\section{Discussion}

In this paper we have presented a detailed report about the adoption and use of a variety of communications technologies among Deaf teenagers. We have answered questions about who they communicate with, what they use to communicate, and where and when they communicate. In answering these questions, we have raised many more, but two points bear further discussion here: first, the teens' use-centric view of mobile computing, and secondly the social acceptability of their chosen device and the tension arising from that choice.

The students' use and understanding of electronic communication was very "use-centric." While not understanding the specifics of the communication mode, they were still able to use the methods. For example, very few students could articulate the differences between text messaging and instant messaging. Instead, they characterized them based on the reply or response time. When asked what text messaging was, many of them responded from a use-centric perspective. Text messaging is "pretty fast" and "you're kinda talking." "...You kinda write a long thing out and then you send it and then you wait a minute and it comes back." This use-centric perspective extended beyond just the differences between text-messaging and instant messaging. When asked about a specific method of 
communication, the students responded first with the characteristics of its usage or with a list of whom they could contact by a specific method. However, while they may not have understood the underlying elements of the device, they had no trouble using the device in a variety of different ways. During the course of the study, the teens cited using the Sidekick for voice telephony, email, IM, Internet search, relay, and grammar/spelling checks.

Our study highlights that how technologists classify and distinguish technologies is different from mainstream, public use. Technologies that are drastically different can be unproblematic in use for end-users. This clarity in usage models is key for usability. In our study, the teens viewed the device as something to be used for communication, and seemed to inherently knew what service to use and when to use that particular service.

As discussed earlier, the teens drew from a wide geographical area which often limited their contact with each other. The Sidekick may have succeeded in this community because it is something which helps reduce this distance in a socially acceptable way. This device, unlike relay or a TTY, is practical to the entire population, not just the deaf or those trying to communicate with them. The Sidekick is accepted by both hearing and Deaf teens, and allows the Deaf teens to be similar to their hearing peers and establish a communication link using that similarity.

However, this similarity comes with a price. In some venues educators and linguists have expressed reservations about instant and text messaging. They have highlighted how the English used in computer-mediated communication differs from that used in more normative language [3, 6]. This is a particularly interesting issue given that our population may have problems with their second language of English, especially with structure and grammar. While the students reported using some acronyms and abbreviations, they were more concerned with whether or not the recipients could understand them. However the teens admitted that others sometimes used acronyms or slang that they didn't understand, with one participant hypothesizing, "Sometimes, they make stuff up." It is worth noting that many slang terms popular in text and instant messaging are phonetically based (e.g. "c u l8r") which would present inherent problems to the Deaf teens. By using informal language, the teens are practicing written English, something their teachers usually encourage.

\section{Conclusions}

While this paper cannot conclusively answer many questions about the design of mobile devices or electronic communication tools for the Deaf community, it can point the way to other avenues worthy of further exploration. Given the second language problems that many Deaf individuals encounter, should we be designing English-based (or other written language-based) interfaces? Should more designers explore ASL-based (or other visual language-based) interfaces for greater accessibility? Or, alternatively, do the good features afforded by visual language-based interfaces outweigh the usage problems for Deaf individuals? 
In terms of more general accessibility, what other applications should we as ICT designers and practitioners introduce to help children who are born deaf overcome the language barriers they face?

This paper has begun to explore some of the communication preferences and platforms in the Deaf community. We have presented an exploratory study which starts to chart the space of electronic communication use by Deaf teens in the North Georgia and Atlanta areas.

We have used qualitative methods such as social mapping, diary studies, and interviews in order to spend time with the teens and learn about them, their needs, and their communication choices. We looked for communication similarities and differences with hearing teenagers and have found both. The five communication themes of Identity, Connection, Control, Tension, and Convenience help inform our design of "use-centric" technologies. We have found similarities in the motivation and reasons that teenagers' desire and want mobile communication technology. But we have also found differences, particularly in how different communication technologies are distinguished from one another.

This paper and the related work are the first steps toward understanding how to design accessible technology for Deaf teenagers. The challenge will be to design technology which simultaneously provides similarities to the mainstream in desirable ways and yet supports cultural differences.

Acknowledgments. Special thanks to Mary Anne Mullins and Harley Hamilton at AASD as well as the teenagers who participated. This work is funded by the NSF (Grants \#0093291, \#0511900, \#0611519, and a Graduate Fellowship) and the US Dept. of Education (Grant \#H133E010804). The opinions and conclusions of this publication do not necessarily reflect those of the NSF or the US DoEd.

\section{References}

1. Bakken, F.: The Inside Text, chapter, SMS Use Among Deaf Teens and Young Adults in Norway, pp. 161-174. Kluwer Academic Publishers, Boston, MA (2004)

2. Barkhuus, L., Vallgårarda, A.: Saying it all in 160 characters: Four classes of SMS conversations. Technical Report TR-45, IT University of Copenhagen, Cophenhagen, Denmark (April 2004)

3. Baron, N.S.: Instant messaging and the future of language. Communications of the ACM 48(7), 29-31 (2005)

4. Baron, N.S., Squires, L., Tench, S., Thompson, M.: Tethered or mobile? Use of away messages in instant messaging by american college students. In: Front Stage - Back Stage: Mobile Communication and the Renegotiation of hte Social Sphere, Grimstead, Norway, June 2003, Springer, Heidelberg (2003)

5. Blinkoff, R., Barranca, M.: Mobile lifestyles. Receiver Magazine 6, Vodafone Group (2002)

6. Crystal, D.: Language and the Internet. Cambridge University Press, Cambridge (2001)

7. Grinter, R., Eldridge, M.: y do tngrs luv 2 txt msg? In: Proc. of the Seventh ECSCW, pp. 219-238 (2001) 
8. Grinter, R., Eldridge, M.: Wan2tlk?: everyday text messaging. In: Proc. of CHI, April 2003, pp. 441-448. ACM Press, New York (2003)

9. Grinter, R.E., Palen, L.: Instant messaging and teen life. In: Proceedings of CSCW '02, New Orleans, LA, November 2002, pp. 21-30 (2002)

10. Harkins, J., Bakke, M.: Oxford Handbook of Deaf Studies, Language, and Education. In: ch. Technologies for Communication: Status and Trends, pp. 407-419. Oxford University Press, Oxford (2003)

11. Holt, J., Traxler, C., Allen, T.: Interpreting the scores: A user's guide to the 9th edition stanford achievement test for educators of deaf and hard-of-hearing students. Gallaudet Research Institute Technical Report 97-1, Gallaudet University, Washington, DC (1997)

12. Lenhart, A., Madden, M., Hitlin, P.: Teens and technology. Technical report, Pew Internet and American Life Project, Washington, DC (July 2005)

13. Ling, R.: We release them little by little: Maturation and gender identity as seen in the use of mobile telephony. Personal Ubiquitous Comput. 5(2), 123-136 (2001)

14. Lofland, J., Lofland, L.H.: Analyzing Social Settings: A Guide to Qualitative Observation and Analysis. Wadsworth Publishing (1995)

15. Mayberry, R.: The critical period for language acquisition and the deaf child's language comprehension: A psycholinguistic approach. Bulletin d'Audiophonologie: Annales Scientifiques de L'Universite de Franche-Comte 15, 349-358 (1998)

16. Nardi, B., Whittaker, S., Bradner, E.: Interaction and outeraction: Instant messaging in action. In: Proceedings of CSCW'00, Philadelphia, PA, pp. 79-88 (2000)

17. Newport, E.: Maturational constraints on language learning. Cognitive Science 14(1), 11-28 (1990)

18. Power, M.R., Power, D.: Everyone here speaks TXT: Deaf people using SMS in Australia and the rest of the world. Journal of Deaf Studies and Deaf Education 9(3), 333-343 (2004)

19. Schiano, D.J., Chen, C.P., Isaacs, E., Ginsberg, J., Gretarsdottir, U., Huddleston, M.: Teen use of messaging media. In: CHI '02. Extended abstracts on Human factors in computing systems, pp. 594-595. ACM Press, New York (2002)

20. Smith, H., Rodgers, Y., Brady, M.: Managing one's social network: Does age make a difference? In: Proceedings of INTERACT '03, pp. 551-558 (2003)

21. Spencer, P., Lederberg, A.: Communication and Language: Discoveries from Atypical Development. In: Different Modes, Different Models: Communication and Language of Young Deaf Children and their Mothers, pp. 203-230. Harvard University Press, Cambridge (1997)

22. Valli, C., Lucas, C.: Linguistics of American Sign Language. Gallaudet University Press, Washington DC (1992)

23. Wagreich, B.: Electronic mail for the hearing impaired and its potential for other disabilities. IEEE Transactions on Communications COM-30(1), 58-65 (1982) 